\title{
'Misconduct' definitions still prove elusive
}

Washington. The most notable aspect of a two-day meeting on scientific conduct last week in Washington, DC, was a striking sense of déjà vu. After more than a decade of intense debate, government rule-making, and at least a dozen reports from professional groups throughout the United States, the meeting remained dominated by the lament that scientific misconduct is hard to define, and even harder to deal with.

The meeting was arranged by the US National Academy of Sciences, the National Academy of Engineering and the Institute of Medicine. One major issue raised was the need for a uniform - and uniformly agreed - definition of misconduct.

The US Public Health Service (PHS), whose rules govern the National Institutes of Health (NIH), operates under a strict definition that includes fabrication, falsification and plagiarism, often referred to as 'FFP'. The PHS also follows a controversial procedure for responding to allegations of misconduct in which the officials conducting the investigation also adjudicate.

By contrast, the National Science Foundation (NSF) separates the investigation from adjudication, but uses an expanded definition of misconduct that includes not only FFP but also "other practices that seriously deviate from those commonly accepted in the scientific community".

The argument against the NSF definition was put persuasively by Howard Schachman of the University of California at Berkeley, recently appointed as integrity ombudsman to the director of NIH.

Schachman said that the federal government, with its power to ban scientists from the research establishment by denying them jobs or grants, should become involved only in potentially criminal offences. The fabrication of data in a grant application, for example, would constitute an attempt to defraud the government.

Others claimed that the research community should be concerned with so-called deviations from accepted practice, and thus favour the NSF's definition. But if an example cited by Neal Lane, the NSF director, offers any insight - namely the secret switching of reagents in a colleague's laboratory - in practice the agency follows a strict definition of "serious deviation".

The core issue faced by the meeting was how to ensure, within the highly competitive environment of modern science, adherence to the standards of ethical behaviour and scientific etiquette that are said to have characterized a previous era.

Authorship practices were one example of the dilemma that received close attention.

\section{ESO seeks new promises from Chile}

Munich. The European Southern Observatory (ESO) has a summer of hard bargaining ahead if its DM500 million (US\$330 million) Very Large Telescope (VLT) is to be installed, as planned, on Mount Paranal in northern Chile.

The site for the telescope has already been levelled (right). And last month an ESO delegation was reassured by the Chilean foreign minister, Carlos Figueroa, that the Chilean government stands by its contractual agreements, dating back to the early 1960 s, guaranteeing ESO privileges that include immunity from local law.

This should mean that ESO is able to ignore recent claims to ownership of the VLT land by a local family, which has disrupted building work and at one point threatened the VLT's future at Mount Paranal (see Nature 368, 676; 1994).

But ESO remains concerned. A statement issued by the organization last week, after the delegation had reported back to the ESO council, said that "unless a clarification of this problem is achieved as soon

\section{IMAGE UNAVAILABLE FOR COPYRIGHT REASONS}

ain: already levelled to receive the VLT.

as possible, it is unlikely that the current plan for the construction of the VLT observatory at Paranal can be maintained."

In particular, ESO wants further reassurance from the courts, which have not always given the same interpretation as the government of ESO's legal status in Chile, before it finally decides to go ahead with the planned shipment of the first VLT building. An extraordinary meeting of the ESO council early in August will reassess the situation.

ESO is adding pressure for an early resolution of the dispute by refusing to sign a lengthily negotiated "supplementary treaty" which, among other things, offers privileges to Chilean astronomers. A. A.
Nearly everyone present agreed that honorary authorship does not promote high ethical standards. But no one could agree just what the proper requirements for authorship should be.

The meeting was therefore left with unanswered questions. For example, if a laboratory director provides space, support and equipment to a student who is not working on one of the director's own projects, should the director still appear as an author on subsequent publications?

Despite efforts by various groups to establish guidelines for recording and maintaining data, this issue also provoked discussion. How should notebooks be kept? Who owns the data? And who should have access to them?

There are usually official answers to such questions. Universities, for instance, tend to be the legal owners of data, as it is the university, not the scientist, that usually receives a grant. But the consensus among the participants was that the area remains a source of confusion.

Not surprisingly, the responsibilities of laboratory heads as mentors also provoked lively discussion. A theme running through the meeting was that the scientific community should hold itself to higher standards of conduct than those written into law. But ways of elaborating and monitoring those higher standards escaped resolution.

That in itself proved to be an interesting (and worrying) observation in the light of the NIH's requirement for universities to give ethics courses to grant recipients, and that the teaching of scientific ethics and etiquette generally has become quite common over the past five years.

Modification of the pressure to publish or perish was put forward by some as an obvious solution. But others felt that, until university tenure committees abandon the practice of evaluating candidates on the basis of quantity rather than quality, it will remain a good but useless adjuration.

Kenneth Ryan of Harvard Medical School (and also chair of the new ethics advisory committee within the Department of Health and Human Services) asked whether either the academy or the Institute of Medicine had mechanisms for expelling members who violate ethical practices.

The answer was that neither does. Indeed, exemplary behaviour is not even a criterion for election to membership of the academy, or indeed of most honorary professional societies.

Bruce Alberts, president of the academy, is faced with digesting the many views expressed at the meeting, and deciding how the academy should lead the way out of the morass of 'scientific integrity' or 'good scientific conduct'. Barbara J. Culliton 